\title{
Reacción alérgica a ciprofloxacina: Descripción de un caso clínico
}

\author{
Eduardo Contreras Z., Sandra X. Zuluaga M., Juan C. Díaz M. y Gloria L. Vejarano J.
}

Fundación Valle del Lili, Cali, Colombia (ECZ, JCDM, GLVJ) Santa Casa da Misericórdia do Rio de Janeiro, Brasil (SXZM)

Recibido: 5 diciembre 2007 Aceptado: 16 septiembre 2008

Correspondencia a: Eduardo Contreras Zúñiga edo11@hotmail.com

\section{Introducción}

$\mathrm{L}$ os efectos secundarios de los fármacos son uno de los mayores problemas de la terapia medicamentosa, la mayor parte de estos efectos secundarios se relacionan con la actividad farmacológica del mismo, pero alrededor de $15 \%$ de estos efectos secundarios se atribuyen a mecanismos inmunológicos.

\section{Caso clínico}

Paciente de sexo femenino, con 24 años de edad, quien consultó por tres días de malestar general, dolor tipo cólico en el hemi-abdomen inferior, disuria, poliaquiria, náuseas, vómitos y escalofríos. No refería dolor lumbar ni fiebre. Ingresó en regulares condiciones generales, deshidratada. PA 90/60 mmHg, FC 118' FR 22'. El resto del examen físico se describió dentro de límites normales. Traía un informe de urocultivo, previamente solicitado, positivo con $>100.000$ ufc de Escherichia coli sensible a multiples antimicrobianos, entre ellos quinolonas. Se inició rehidratación y se le solicitaron exámenes complementario comprobándose una acidosis metabolica, leucocitosis y neutrofilia, creatininemia de $1,2 \mathrm{mg} / \mathrm{dl}$ y electrolitos normales. El uro-análisis fue compatible con infección urinaria. Dado estos hallazgos clínicos y de laboratorio, fue hospitalizada para hidratación y antibioterapia intravenosa, indicándosele ciprofloxacina $400 \mathrm{mg}$ iv cada 12 horas. Experimentó una evolución lenta hacia la mejoria pero, tras 36 horas de iniciado el antimicrobiano presentó un rash máculo papular generalizado, pruriginoso (Figura 1) y deterio- ro del estado de conciencia, asociado a un episodio convulsivo. Se suspendió la ciprofloxacina, se administró un antihistamínico y corticosteroides iv y se procedió a realizar una TAC cerebral, la cual fue normal. La paciente evolucionó satisfactoriamente tras el cambio de antimicrobiano a ceftriaxona. Fue dada de alta al $7^{\circ}$ día de haber ingresado.

Para obtener un diagnóstico más exacto, se la realizó un prick-test cuatro semanas más tarde, siendo éste positivo con un halo de $5 \mathrm{~mm}$ mayor al control. Además, se le practicó una prueba de provocación con una exposición oral a dosis bajas $(250 \mathrm{mg})$ de ciprofloxacina durante la cual presentó una reacción alérgica con prurito, tos y disnea.

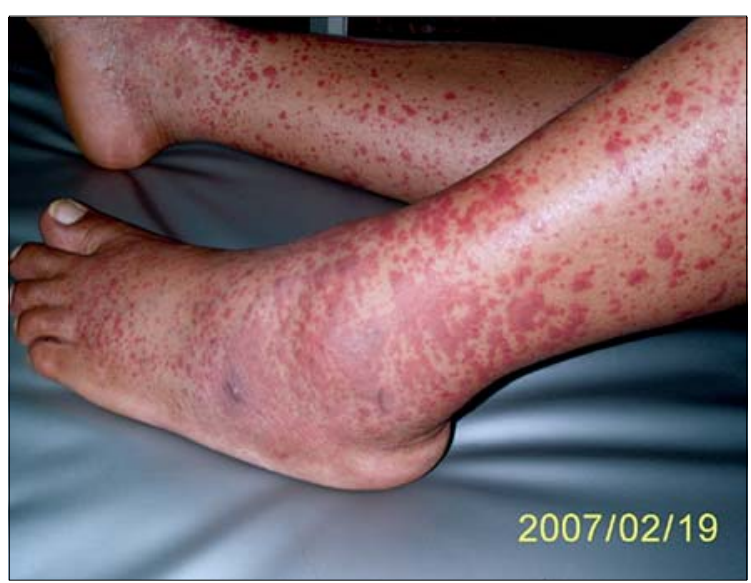

Figura 1. Lesiones máculo papulares pruriginosas, generalizadas, de aspecto vasculítico que desaparecen progresivamente tras la suspensión de la quinolona. 


\section{Discusión}

Clásicamente se utiliza la clasificación de Gell y Coobs para explicar los mecanismos responsables de las alteraciones patológicas inducidas por fármacos: el tipo I mediado por IgE, el tipo II citotóxico, el tipo III mediado por inmunocomplejos y el tipo IV mediado por células. En el caso de las quinolonas la reacción de hipersensibilidad es principalmente de tipo $\mathrm{I}^{1,2}$.

Las quinolonas integran una familia de antibacterianos conocida desde la década del 60, a partir de la investigación de antimaláricos. La primer quinolona usada en clínica fue el ácido nalidíxico, introducido en $1962^{3}$.

Desde entonces, se han sintetizado e investigado gran número de quinolonas, buscando incrementar su actividad y espectro de acción y reducir sus efectos $\operatorname{adversos}^{3,4}$.

En general son bien toleradas, con un perfil de seguridad similar para todos los componentes del grupo. Las reacciones alérgicas a quinolonas son infrecuentes y, en su mayoría, las manifestaciones son leves y retroceden al suspender el medicamento ${ }^{5-7}$.

Los efectos adversos más frecuentes son alteraciones gastrointestinales, seguidos de síntomas neuropsiquiátricos y de reacciones cutáneas de hipersensibilidad $^{3,8}$.

Como manifestación gastrointestinal pueden observarse náuseas, vómitos, diarrea, dolor abdominal, pérdida del apetito y malestar abdominal. La colitis por Clostridium difficile es infrecuente ${ }^{6}$.

Entre las manifestaciones adversas neurológicas más frecuentes se describen mareo, cefalea, insomnio y alucinaciones. Se destacan, por su gravedad, las convulsiones y las reacciones maníacas o psicóticas, especialmente en personas con enfermedades previas del SNC: epilepsia, tumores cerebrales, arterioesclerosis, hipoxia cerebral o alteraciones metabólicas, por lo que se recomienda evitar estos antibacterianos en estos enfermos. El riesgo de convulsiones aumenta con la administración concomitante de algunos fármacos como: antiinflamatorios no esteroidal y teofilina. En este caso, la paciente no tenía antecedentes personales o familiares relevantes ni ingería alguno de los fármacos previamente mencionados pero, aún así, presentó un episodio convulsivo asociado al uso de ciprofloxacina $^{9-11}$.

Las reacciones cutáneas de hipersensiblidad, se caracterizan por rash y prurito. En general, son leves o moderadas y ceden al suspender el fármaco. Se han descrito formas más graves, pero con una baja inci$\operatorname{dencia}^{6,8,9}$.

Ante esta alta sospecha clínica se realizaron a la paciente diversos estudios confirmatorios como fueron:
- Prick test. Consiste en la colocación de una gota de extracto alergénico -en este caso de ciprofloxacinasobre la piel sana del individuo, puncionando a través de dicha gota con una lanceta especial de 1 $\mathrm{mm}$, lo que introduce una parte mínima de dicho extracto en la piel. El prick test detecta la IgE específica ligada a los receptores celulares en la superficie de los mastocitos. De tal modo que, cuando un paciente está sensibilizado a un determinado alergeno, la introducción de dicho alergeno en la zona dérmica repite el proceso de interacción antígenoanticuerpo (alergeno-IgE específica) provocando la degranulación de los mastocitos y la aparición de un habón y un halo eritematoso circundante. El tamaño del habón, evaluado a los 15-20 minutos, determina la magnitud de la reacción. El prick test es positivo si la reacción cutánea al antígeno resulta $3 \mathrm{~mm}$ mayor comparada con el control ${ }^{6,7}$.

- Pruebas de provocación. Consiste en la administración, controlada y gradual, de la sustancia sospechosa a través de diferentes vías: oral, conjuntival, nasal, bronquial, etc, para comprobar su tolerancia. Se usa siempre que el estudio diagnóstico previo mediante pruebas cutáneas y determinación de $\operatorname{IgE}$ específica sérica (según casos) muestre resultados negativos, no concluyentes, o bien no se ha podido llevar a cabo, por el motivo que sea. No distingue entre alergia e intolerancia ${ }^{7,8}$.

En esta paciente, tanto el prick test como las pruebas de provocación fueron positivas, confirmándose la sospecha diagnóstica de reacción alérgica a ciprofloxacina ${ }^{2,10}$.

Se han descrito además reacciones de fotosensibilidad con sparfloxacina, lomefloxacina y enoxacina, por lo que debe evitarse la exposición al sol hasta cinco días después de discontinuado el fármaco ${ }^{4,10}$.

En pacientes que presentan una reacción alérgica aguda a alguna quinolona, debe prescribirse un antimicrobiano de otro grupo, dado que existe hipersensiblidad cruzada entre las diferentes quinolonas. En el caso expuesto, con la administración consecuente de ceftriaxona, la paciente no presentó reacción adversa alguna.

\section{Resumen}

Las reacciones alérgicas a quinolonas son infrecuentes. Se describe a continuación el caso clínico de una paciente con una reacción cutánea y sistémica grave a ciprofloxacina, posterior a su indicación por una infección del tracto urinario. Para obtener un diagnóstico más exacto, se realizó un Prick-test el 
cual fue positivo, así como pruebas de provocación con una exposición oral a dosis bajas. En la prueba de provocación efectuada con $250 \mathrm{mg}$ de ciprofloxacina oral, presentó prurito, tos y disnea. Ambas pruebas sugieren, en este caso, un mecanismo mediado por $\operatorname{IgE}$.

\section{Referencias}

1.- Luskin A T, Luskin S S. Anaphylaxis and anaphylactoid reactions: diagnosis and management. Am J Ther 1996; 3: 515-20.

2.- Schmid D A, Campi P, Pichler W J. Hypersensitivity reactions to quinolones. Curr Pharm Des 2006; 12: 3313-26.

3.- Schmid D A, Depta J P, Pichler W J. $\mathrm{T}$ cell-mediated hypersensitivity to quinolones: mechanisms and crossreactivity. Clin Exp Allergy 2006; 36: 59-69.

4.- Sanders W E. Efficacy, safety, and potential economic benefits of oral ciprofloxacin in the treatment of infections. Rev Infect Dis 1988; 10: 528-43.

5.- Copioli J, Arab D, Bornancini M, Barrera R, Abarca R. Hipersensibilidad a quinolonas: presentación de casos. Alerg Inmunol Clin 2005; 23: 31-5.

6.- Venturini Díaz M, Lobera Labairu T, del Pozo Gil MD, Blasco A. In vivo diagnostic tests in adverse reactions to quinolones. $\mathrm{J}$ Investig Allergol Clin Immunol 2007; 17: 393-8.

7.- Messaad D, Sahla H, Benahmed S, Godard P. Drug provocation tests in patients with a history suggesting an immediate drug hypersensitivity reaction. Ann Intern Med
2004; 140: 1001-6.

8.- Lammintausta K, Kortekangas-Savolainen O. The usefulness of skin tests to prove drug hypersensitivity. Br J Dermatol 2005; 152: 968-74.

9.- Campi P. Quinolone hypersensitivity. Curr Opin Allergy Clin Immunol 2003; 3: 275-81.

10.- González I, Lobera T, Blasco A, del Pozo M D. Immediate hypersensitivity to quinolones: moxifloxacin crossreactivity. J Investig Allergol Clin Immunol 2005; 15: 146-9.

11.- Scherer K, Bircher A J. Hypersensitivity reactions to fluoroquinolones. Curr Allergy Asthma Rep 2005; 5: 15-21. 